\title{
Exposition radiologique de l'équipe opératoire au cours de curiethérapies de prostate par implants permanents d'iode-125
}

\author{
G. GAGNA ${ }^{1}$, C. GAURON ${ }^{2}$, J.-C. AMABILE ${ }^{1}$, P. LAROCHE ${ }^{1}$
}

(Manuscrit reçu le 22 décembre 2010, accepté le 12 février 2010)

RÉSUMÉ Le Service de protection radiologique des armées (SPRA) et l'Institut national de recherche et de sécurité (INRS) ont mené conjointement une étude afin d'évaluer l'exposition radiologique de l'équipe opératoire au cours de curiethérapies de prostate par implants permanents d'iode-125 à l'Hôpital d'instruction des armées du Val-de-Grâce. Le but de cette étude est d'estimer les doses reçues par une équipe nouvellement formée à cette technique, de mesurer les différents débits d'équivalent de dose ambiant et de définir le zonage radiologique. Au cours des six séances de curiethérapie étudiées, les doses efficaces enregistrées par des dosimètres passifs OSL InLight $^{\circledR}$ corps entier et les doses équivalentes aux extrémités et au cristallin mesurées par des dosimètres nanoDot ${ }^{\circledR}$ sont restées inférieures au seuil de détection pour l'ensemble des personnels. Le débit d'équivalent de dose ambiant mesuré en fin d'implantation par un radiamètre $A T 1123{ }^{\circledR}$ est en moyenne de $170 \mu \mathrm{Sv} / \mathrm{h}$ au niveau du périnée du patient. La limite théorique de la zone contrôlée est estimée à environ $20 \mathrm{~cm}$ du périnée du patient. À partir de ces résultats, les auteurs proposent des recommandations concernant la catégorisation, le zonage radiologique et les modalités de surveillance dosimétrique des personnels. La curiethérapie de prostate par voie transpérinéale échoguidée en temps réel est une technique peu irradiante pour le personnel du fait des caractéristiques de la source radioactive et des moyens techniques utilisés qui assurent une radioprotection efficace de l'équipe opératoire.

ABSTRACT Radiation exposure to operating room staff during prostate brachytherapy using iodine-125 seeds.

The French defense radiation protection service (SPRA) and the French national institute for research and safety (INRS) conducted a joint study to assess the radiation exposure to operating room staff during prostate brachytherapy using iodine-125 seeds at the Val-de-Grâce military hospital. The purpose of the study was the assessment of the effective doses, the equivalent doses to the extremities and lens received by a novice team, the different ambient dose equivalent rates measurements and the delineation of areas. After six brachytherapies, all the recorded doses with

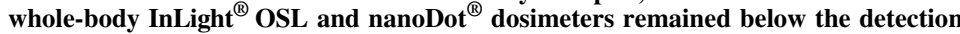
limit for the whole staff. The dose rate measured at the end of implantation by an $\mathrm{AT}_{1123}{ }^{\circledR}$ survey meter is about $170 \mu \mathrm{Sv} / \mathrm{h}$ at the perineum of the patient. The controlled area limit is estimated to be about $20 \mathrm{~cm}$ from the patient perineum. From these results, the authors propose recommendations for the categorization of workers, the delineation of areas and the dose monitoring procedures. This study demonstrates that real-time ultrasound-guided transperineal prostate brachytherapy delivers low dose to the operators because of the radioactive source characteristics and the instrumentation providing an effective radiation protection for the surgical team.

Keywords: Brachytherapy / dosimetry personel / ${ }^{125}$ I iodine / prostate cancer / radiation exposure

\footnotetext{
1 Service de protection radiologique des armées (SPRA), 1 bis rue du Lieutenant Raoul Batany, 92141 Clamart Cedex, France.

2 Institut national de recherche et de sécurité (INRS), Département Études et Assistance Médicales, 30 rue Olivier Noyer, 75680 Paris Cedex 14, France.
} 


\section{Introduction}

La prise en charge du cancer de la prostate à un stade localisé repose sur différentes alternatives thérapeutiques (prostatectomie radicale, radiothérapie externe ou curiethérapie) aux résultats comparables en termes de contrôle tumoral. Ces différentes techniques sont proposées au patient en fonction de critères bien établis. Parmi elles, la curiethérapie de prostate par implantation permanente de grains d'iode-125 sous contrôle échographique est aujourd'hui une option thérapeutique validée. Cette technique est de plus en plus utilisée du fait du dépistage précoce de ces tumeurs et des moindres complications en termes d'incontinence et de préservation de la fonction sexuelle (Cosset et al., 2004).

Développée dans de nombreux centres en France, la curiethérapie de prostate est proposée depuis décembre 2009 à l'Hôpital d'instruction des armées (HIA) du Val-de-Grâce. Les premières séances ont débuté en avril 2010 après une première phase d'apprentissage auprès d'un radiothérapeute expérimenté extérieur à l'établissement.

Bien que cette technique soit reconnue par bon nombre de spécialistes comme peu irradiante pour l'équipe opératoire (ICRP, 2005), les publications concernant l'exposition radiologique de ces personnels restent rares dans la littérature scientifique. Il nous est donc apparu pertinent d'évaluer, par une méthodologie rigoureuse et des moyens techniques adaptés, les doses reçues par des professionnels peu expérimentés et de mesurer l'ambiance radiologique au bloc opératoire.

Cette étude dosimétrique est menée de mai à août 2010 au bloc opératoire de l'HIA du Val-de-Grâce au cours de six séances de curiethérapie par les équipes techniques du Service de protection radiologique des armées (SPRA), en partenariat avec l'Institut national de recherche et de sécurité (INRS).

\section{Matériels et méthodes}

\subsection{Déroulement de l'intervention}

\subsubsection{Description de la technique}

L'intervention est réalisée au bloc opératoire, sous anesthésie générale. Le patient est en position de la taille (décubitus dorsal, hanches et genoux fléchis). Elle fait intervenir une équipe pluridisciplinaire comprenant un radiothérapeute, un urologue, un radiophysicien ainsi qu'une équipe opératoire classique : médecin anesthésiste, infirmier anesthésiste diplômé d'état (IADE) et infirmier de bloc opératoire diplômé d'état (IBODE). 
Cette technique, dite en temps réel, se déroule en un seul temps (Cosset et Haie-Meder, 2005). Le volume prostatique est mesuré par échographie endorectale permettant la réalisation d'une dosimétrie prévisionnelle et la détermination du nombre de grains d'iode à implanter.

L'urologue implante d'abord, par voie transpérinéale et sous contrôle échographique, les aiguilles vectrices en périphérie de la glande à travers une grille de repérage (Fig. 1). Le temps radiologique de l'intervention débute par l'implantation, par le radiothérapeute, d'environ $75 \%$ des grains dans cette région à travers ces aiguilles vectrices (Fig. 2).

Le positionnement exact des grains est visualisé sur une reconstruction tridimensionnelle des images échographiques qui permet au radiophysicien de déterminer en temps réel, au moyen de courbes dose-volume, la répartition de la dose délivrée à la prostate et aux organes sensibles avoisinants (urètre et rectum). Ce résultat dosimétrique intermédiaire permet une évaluation prévisionnelle du positionnement des grains centraux (à la base et l'apex de la prostate) afin d'optimiser la distribution de la dose.

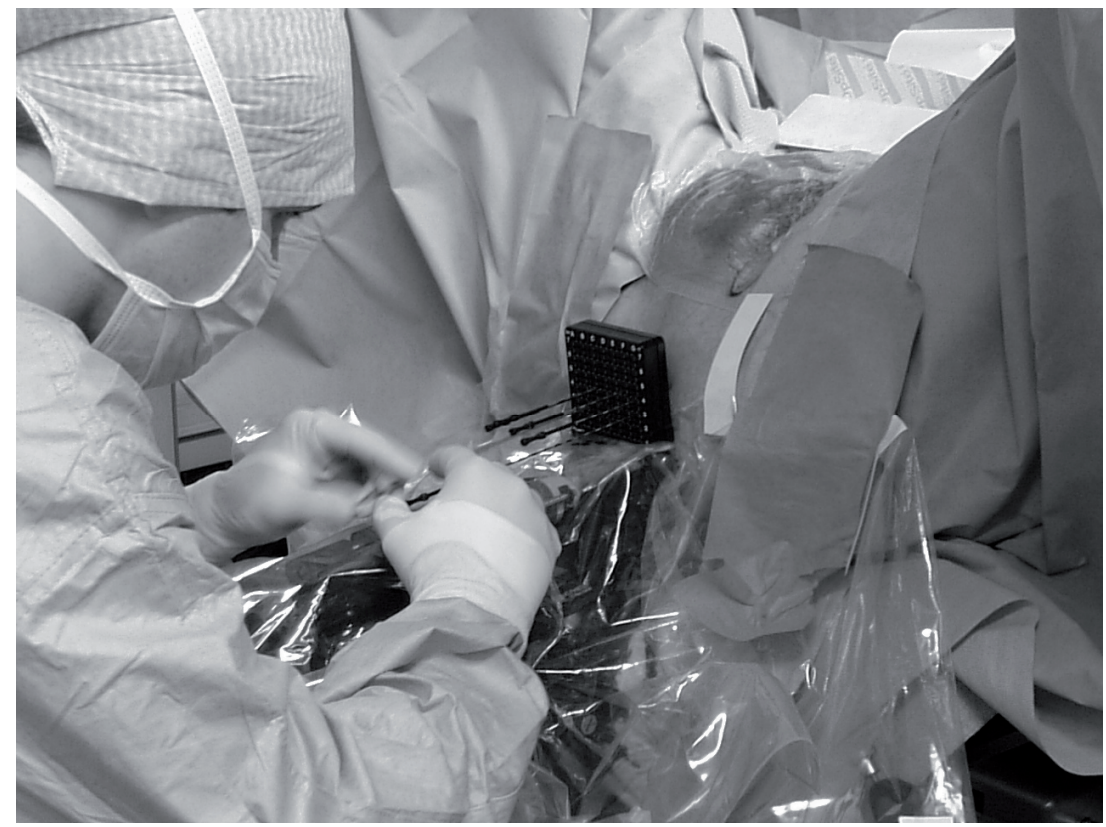

Figure 1 - Implantation des aiguilles vectrices par l'urologue.

Implantation of implant needles by the urologist. 


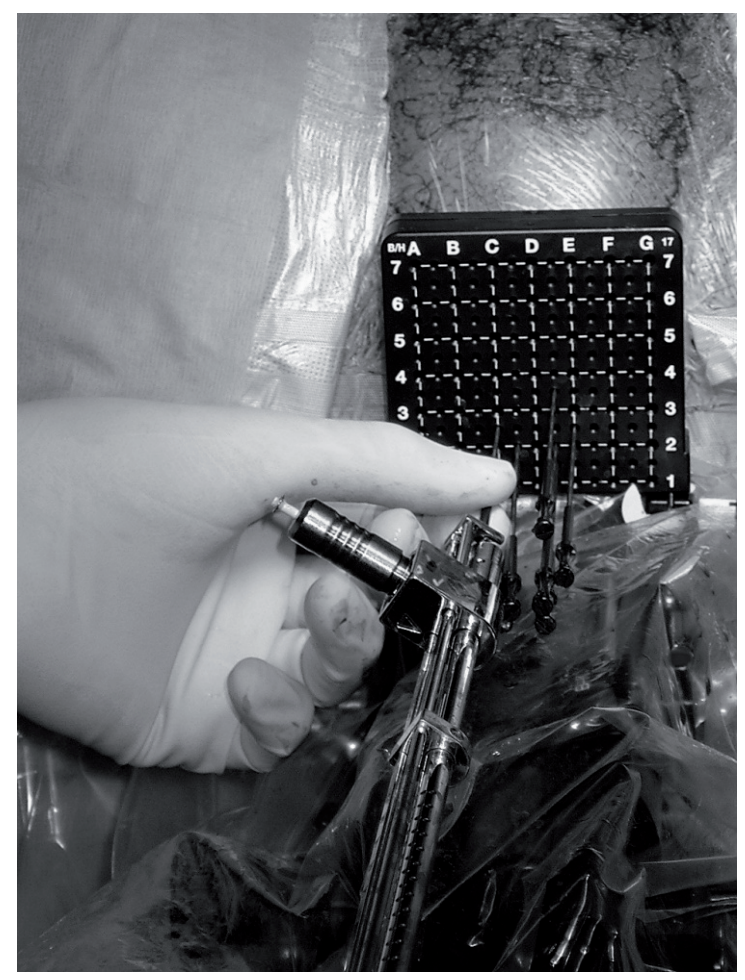

Figure 2 - Implantation des grains d'iode par le radiothérapeute.

Implantation of iodine seeds by the radiotherapist.

Les aiguilles vectrices centrales sont alors implantées par l'urologue, puis les grains centraux sont implantés par le radiothérapeute selon la géométrie prévue par ce plan.

En fin d'intervention, un cliché radiographique du pelvis de face permet de contrôler que la répartition des grains d'iode au sein de la prostate est homogène.

\subsubsection{Description des sources radioactives}

Les grains d'iode-125 mesurent 4 à $5 \mathrm{~mm}$ de long, moins de $1 \mathrm{~mm}$ de diamètre et sont enrobés d'une capsule de titane (Peiffert, 2005). La période radioactive de l'iode-125 est d'environ 60 jours et l'activité de chaque grain est proche de $18,5 \mathrm{MBq}(0,5 \mathrm{mCi})$. L'énergie des photons émis est faible (27 à $35 \mathrm{keV})$. Ces grains sont stockés dans des cartouches pré-chargées en acier de 15 grains maximum. Les grains libres sont implantés à l'unité par le radiothérapeute au moyen d'un 
projecteur de source, le « Mick applicator ${ }^{\circledR}$ », et délivrent à la glande une irradiation permanente à bas débit de dose ( 0,4 à $2 \mathrm{~Gy} / \mathrm{h}$ au volume cible) .

Les données générales de l'intervention: activité administrée et nombre de grains implantés, durée totale et temps «radiologique» (après ouverture des sources) de l'intervention, temps d'exposition de l'urologue (mise en place des aiguilles centrales) et du radiothérapeute (implantation des grains périphériques et centraux) sont évaluées au cours de chaque séance de curiethérapie.

\section{2. Évaluation de la dose reçue par les personnels}

\subsection{1. Évaluation de la dose efficace}

Dans le cadre de cette étude, tous les membres de l'équipe opératoire (urologue, radiothérapeute, radiophysicien, IBODE et IADE) portent lors de chaque séance de curiethérapie :

- un dosimètre passif OSL (optical stimulation luminescence) InLight ${ }^{\circledR}$ (société Landauer), utilisé par le ministère de la défense pour la surveillance de l'exposition externe des travailleurs aux rayonnements ionisants. Ce dosimètre, porté au niveau de la poitrine, est sensible aux rayonnements $\gamma$ et $X$ de $5 \mathrm{keV}$ à $40 \mathrm{MeV}$, avec un seuil de détection théorique de $0,05 \mathrm{mSv}(0,10 \mathrm{mSv}$ en pratique compte tenu de contraintes techniques de lecture du laboratoire de dosimétrie). La grandeur mesurée par ce dosimètre est l'équivalent de dose individuel en profondeur à $10 \mathrm{~mm} \mathrm{Hp} \mathrm{(10),} \mathrm{estimateur} \mathrm{de} \mathrm{la} \mathrm{dose} \mathrm{efficace} \mathrm{à} \mathrm{l'organisme} \mathrm{entier.}$ Les dosimètres ont été lus par le laboratoire de dosimétrie du SPRA après chaque séance de curiethérapie puis, sans avoir effacé la dose, réattribués au même intervenant pour la séance suivante, permettant ainsi un cumul de la dose efficace reçue.

- un dosimètre opérationnel électronique DMC $2000 \mathrm{X}^{\circledR}$ (société MGP instruments), utilisé par les équipes médicales au bloc opératoire de l'HIA du Val-de-Grâce. Porté lui-aussi au niveau de la poitrine, ce dosimètre est adapté à la détection des photons de $20 \mathrm{keV}$ à $6 \mathrm{MeV}$, pour un débit de dose s'étalant de $0,1 \mu \mathrm{Sv} / \mathrm{h}$ à $10 \mu \mathrm{Sv} / \mathrm{h}$ et avec un seuil de détection théorique de $1 \mu \mathrm{Sv}$. La grandeur mesurée est également l'équivalent de dose individuel en profondeur Hp (10). Le dosimètre électronique intégrant et cumulant les doses efficaces reçues, les doses enregistrées sont relevées après chaque curiethérapie.

\subsection{2. Évaluation de la dose équivalente aux extrémités}

La dose reçue au niveau des extrémités a été évaluée pour les opérateurs (radiothérapeute et urologue). Pour cette mesure, nous avons utilisé des dosimètres 
de type nanoDot ${ }^{\circledR}$ (société Landauer) qui sont des dosimètres OSL sous forme de pastille de $4 \mathrm{~mm}$ de diamètre inséré dans une fine plaque de moins de $1 \mathrm{~cm}$ de côté, dont la réponse en énergie de $5 \mathrm{keV}$ à $40 \mathrm{MeV}$ est adaptée aux basses énergies des photons de l'iode-125. Le seuil de détection de ces dosimètres est lui aussi de $0,1 \mathrm{mSv}$ en pratique (le seuil théorique constructeur est de $0,05 \mathrm{mSv}$ ).

Ces dosimètres ont été fixés avant chaque intervention, après lavage chirurgical des mains, sur l'ongle de l'index gauche du radiothérapeute (Fig. 3) et la pulpe de l'annulaire gauche de l'urologue, au moyen de bandelettes adhésives type StériStrips ${ }^{\circledR}$. Le choix de ces emplacements a été décidé d'une part après l'évaluation préalable des risques qui a montré que le membre non dominant (main gauche en l'occurrence) était le plus exposé, et d'autre part, par le choix des praticiens qui souhaitaient conserver leur dextérité. Les deux dosimètres nanoDot ${ }^{\circledR}$ ont été lus au moyen du lecteur portable microStar ${ }^{\circledR}$ du SPRA après chaque séance de curiethérapie puis, sans avoir effacé la dose, réutilisés par le même intervenant pour la séance suivante, permettant ainsi le cumul de l'équivalent de dose $\mathrm{Hp}(0,07)$ reçue au niveau des extrémités.

\subsection{3. Évaluation de la dose équivalente au cristallin}

L'évaluation préalable des risques a révélé que les yeux de l'urologue et du radiothérapeute étaient relativement près de la grille de repérage. Il nous est alors apparu intéressant d'évaluer la dose équivalente reçue au niveau du cristallin, d'autant plus que les données récentes de la littérature posent la question d'un

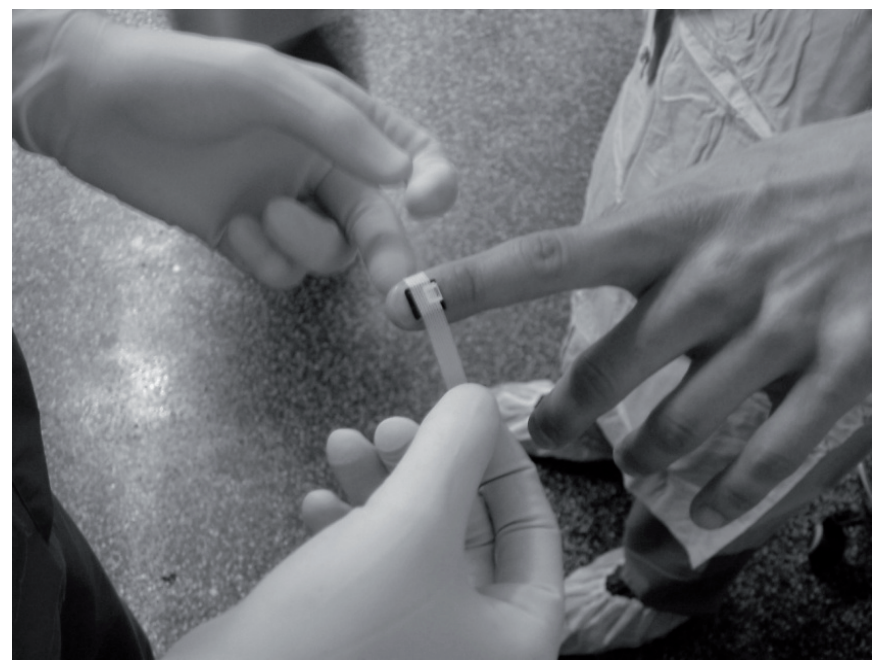

Figure 3 - Dosimètre nanoDot ${ }^{\circledR}$ fixé sur l'index du radiothérapeute.

Position of the nanoDot ${ }^{\circledR}$ dosimeter on the radiotherapist index finger. 
abaissement de cette limite réglementaire (Wassilieff, 2009). La révision de cette limite au cristallin est d'ailleurs actuellement discutée par les experts de la CIPR. Nous avons donc utilisé, selon le même protocole, un dosimètre nanoDot ${ }^{\circledR}$ fixé au moyen d'un film collant transparent type Opsite ${ }^{\circledR}$ sur le front de l'urologue et du radiothérapeute (Fig. 4), pour mesurer l'équivalent de dose $\mathrm{Hp}(0,07)$ reçue à la peau. Nous avons considéré que cette dose mesurée était très proche de la valeur de l'équivalent de dose au cristallin $\mathrm{Hp} \mathrm{(3)} \mathrm{du} \mathrm{fait} \mathrm{du} \mathrm{positionnement} \mathrm{du} \mathrm{dosimètre}$ et de la nature photonique du rayonnement.

\subsubsection{Choix des dosimètres}

Pour l'évaluation de la dose efficace, le choix des dosimètres s'est naturellement porté sur ceux utilisés en pratique courante pour la surveillance des personnels exposés aux rayonnements ionisants du ministère de la défense, ces dosimètres étant théoriquement adaptés aux basses énergies d'émission de l'iode-125.

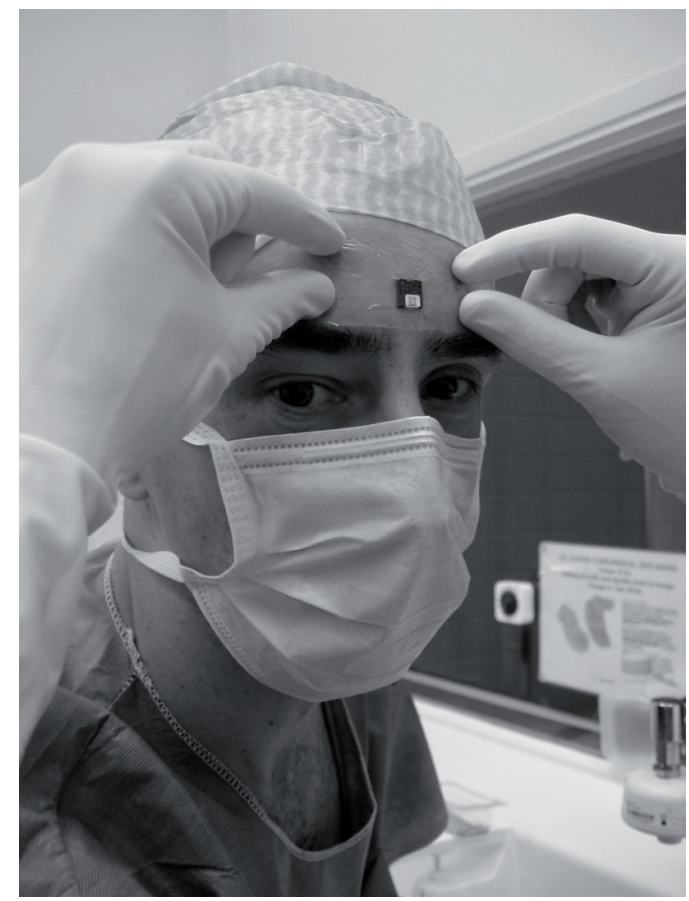

Figure 4 - Dosimètre nanoDot ${ }^{\circledR}$ fixé sur le front du radiothérapeute.

Position of the nanoDot ${ }^{\circledR}$ dosimeter on the radiotherapist front. 
Pour l'évaluation de la dose équivalente aux extrémités, l'emploi de dosimètres nanoDot ${ }^{\circledR}$ nous a semblé plus pertinent par rapport à l'utilisation d'une bague TLD (thermo luminescence dosimeter) en raison :

- de la lecture non destructive de l'information par technologie OSL qui permet une lecture après chaque curiethérapie ;

- de la possibilité d'incrémentation des doses reçues ;

- du gain de distance, donc de dose détectée puisque placés à l'extrémité du doigt (faibles doses attendues).

\section{3. Évaluation de la dosimétrie d'ambiance}

\subsubsection{Caractérisation de l'ambiance radiologique}

Pour la surveillance de zone, la grandeur opérationnelle appropriée est l'équivalent de dose ambiant $\mathrm{H}^{*}(10)$ mesuré au poste de travail par des instruments étalonnés. Cette grandeur est un bon estimateur des grandeurs de protection (dose efficace) et permet la comparaison avec les limites réglementaires.

Pour notre étude, nous avons utilisé un radiamètre AT1123 ${ }^{\circledR}$ (société APVL Ingénierie), adapté pour les mesures dans les champs X et $\gamma$ de $15 \mathrm{keV}$ à $10 \mathrm{MeV}$. Afin d'obtenir une meilleure sensibilité pour les photons de basse énergie, les mesures ont été effectuées sans le capot écran ${ }^{3}$ du détecteur (scintillateur plastique). En pratique, nous avons mesuré les débits d'équivalent de dose ambiant en différents points et temps de l'intervention. La valeur, exprimée en $\mu \mathrm{Sv} / \mathrm{h}$ (multiples ou sous-multiples) est lue directement sur l'écran de l'appareil car le coefficient d'étalonnage est proche de 1 . Ces mesures, réalisées lors de chaque séance par un contrôleur du Bureau de sécurité radiologique du SPRA, ont permis d'évaluer différents débits de dose :

- au contact du porte source ;

- au contact de la cartouche pré-chargée avec protection et grain d'iode à nu ;

- patient sur table, au pseudo-contact du périnée, à $20 \mathrm{~cm}$ et à $1 \mathrm{~m}$ dans différentes directions, après l'implantation des grains périphériques et en fin d'intervention.

\subsubsection{Détermination du zonage radiologique}

Conformément à l'arrêté zonage du 15 mai $2006^{4}$, l'équivalent de dose ambiant est mesuré par l'intégration de la dose enregistrée sur l'heure d'activité la plus

\footnotetext{
3 Méthode de mesure préconisée dans la notice constructeur pour l'évaluation du débit d'équivalent de dose ambiant $\mathrm{H}^{*}$ (10) pour les basses énergies.

4 Rappel des limites réglementaires en dose efficace intégrée $\mathrm{E}_{\mathrm{T}}$ sur la période considérée (le mois ou l'heure) pour les différentes zones :

$\mathrm{E}_{\mathrm{T}}<80 \mu \mathrm{Sv}$ en 1 mois pour la zone non réglementée,

$\mathrm{E}_{\mathrm{T}}<7,5 \mu \mathrm{Sv}$ en 1 heure pour la zone surveillée (ZS),

$\mathrm{E}_{\mathrm{T}}<25 \mu \mathrm{Sv}$ en 1 heure pour la zone contrôlée (ZC).
} 


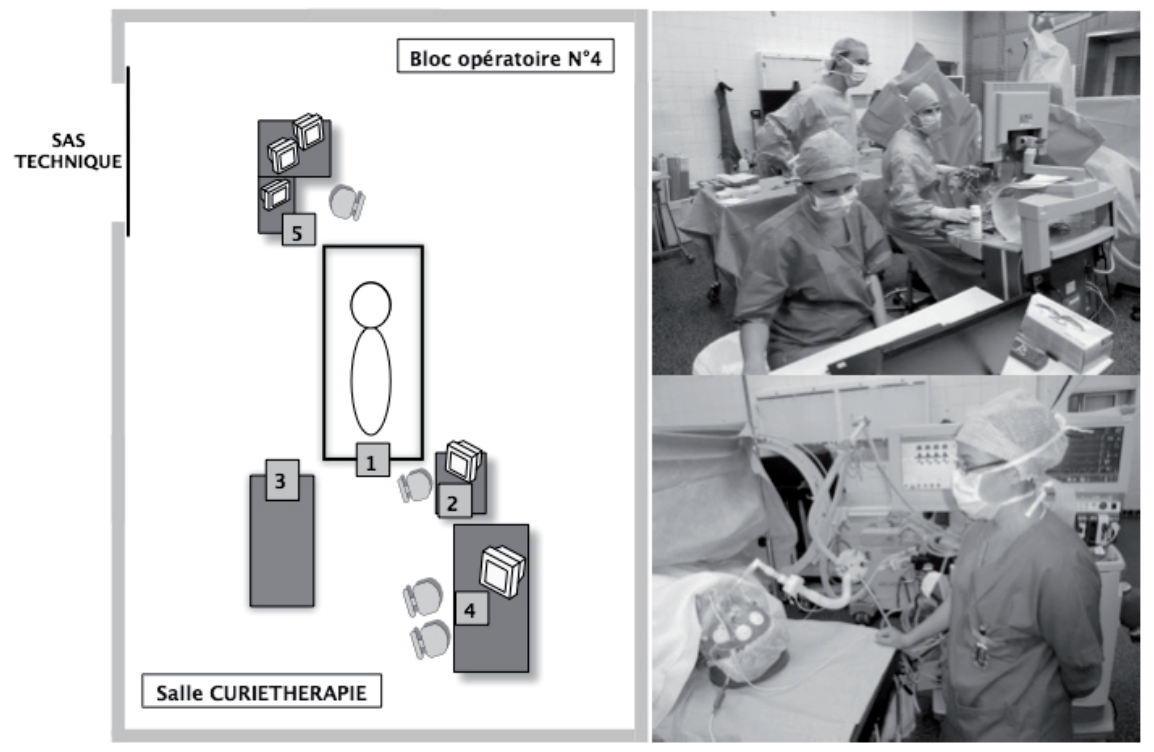

Figure 5 - Représentations schématiques et photographiques des différents postes de travail.

Schematic and photographic representations of the different workstations.

pénalisante (la dernière heure de l'intervention) au moyen d'un radiamètre AT1123 ${ }^{\circledR}$. L'appareil de mesure est ainsi placé, séance après séance, au niveau des différents postes de travail (Fig. 5) :

- poste de travail $\mathrm{n}^{\circ} 1$ dit «opérateur » : radiamètre fixé à environ $30 \mathrm{~cm}$ du périnée (déclenchement à distance par une télécommande) sur la branche reliant le dispositif d'implantation à la table d'opération ;

- poste de travail $\mathrm{n}^{\circ} 2$ : au niveau de l'écran de contrôle de l'échographe pour l'urologue, à $80 \mathrm{~cm}$ du périnée du patient ;

- poste de travail $\mathrm{n}^{\circ} 3$ : table d'instrumentation de l'IBODE, à $50 \mathrm{~cm}$ du périnée du patient ;

- poste de travail $\mathrm{n}^{\circ} 4$ : pupitre du radiophysicien placé à environ $150 \mathrm{~cm}$ du périnée du patient ;

- poste de travail ${ }^{\circ} 5$ : écrans de monitorage de l’IADE, à $160 \mathrm{~cm}$ du périnée du patient.

Le classement des zones au niveau des différents postes de travail sera déterminé sur la base de 40 heures ${ }^{5}$ de travail mensuel (scénario maximaliste).

\footnotetext{
540 heures $=2$ (nombre de curiethérapie/semaine $) \times 2$ (temps moyen en heures estimé d'une curiethérapie $) \times 5($ nombre de semaine majoré dans un mois) × 2 (scénario maximaliste basé sur un doublement de l'activité).
} 
Cette valeur correspond à l'hypothèse enveloppe retenue correspondant à un doublement de l'activité par rapport aux objectifs fixés par l'équipe opératoire de deux curiethérapies par semaine.

La limite de la zone contrôlée sera elle calculée à partir de la dose enregistrée la plus élevée selon la loi de l'inverse du carré de la distance.

\section{RÉSULTATS}

\subsection{Données générales de l'intervention}

Les données générales, synthétisées dans le tableau I, montrent une décroissance régulière des temps d'intervention et d'exposition au cours des six curiethérapies.

\subsection{Dosimétrie des personnels}

\subsubsection{Dose efficace}

Les doses efficaces cumulées au cours des six curiethérapies, par les dosimètres OSL InLight ${ }^{\circledR}$ corps entier portés par l'équipe opératoire, sont restées inférieures au seuil de détection de $100 \mu \mathrm{Sv}$.

Les doses relevées lors de chaque curiethérapie par les dosimètres opérationnels n'ont pas dépassé le seuil de détection de $1 \mu \mathrm{Sv}$ pour l'ensemble des personnels.

\section{TABLEAU I}

Données générales des séances de curiethérapie de prostate.

General prostate brachytherapy data.

\begin{tabular}{|c|c|c|c|c|c|c|c|}
\hline \multicolumn{2}{|c|}{ Séances curiethérapie } & 1 & 2 & 3 & 4 & 5 & 6 \\
\hline \multicolumn{2}{|c|}{$\begin{array}{l}\text { Nombre de grains } \\
\text { d'iode implantés }\end{array}$} & 76 & 72 & 62 & 67 & 61 & 74 \\
\hline \multicolumn{2}{|c|}{$\begin{array}{l}\text { Activité totale administrée } \\
\text { (MBq) }\end{array}$} & 1221 & 1118 & 962 & 1040 & 946 & 1149 \\
\hline \multirow{2}{*}{$\begin{array}{l}\text { Temps } \\
\text { d'intervention }\end{array}$} & Total & $2 \mathrm{~h} 50 \mathrm{~min}$ & $2 \mathrm{~h} 05 \mathrm{~min}$ & $1 \mathrm{~h} 45 \mathrm{~min}$ & $1 \mathrm{~h} 30 \mathrm{~min}$ & $1 \mathrm{~h} 15 \mathrm{~min}$ & $1 \mathrm{~h} 35 \mathrm{~min}$ \\
\hline & Radiologique & $1 \mathrm{~h} 40 \mathrm{~min}$ & $1 \mathrm{~h} 15 \mathrm{~min}$ & $1 \mathrm{~h} 05 \mathrm{~min}$ & $55 \mathrm{~min}$ & $45 \mathrm{~min}$ & $1 \mathrm{~h}$ \\
\hline \multirow{2}{*}{$\begin{array}{l}\text { Temps } \\
\text { d'exposition }\end{array}$} & $\begin{array}{l}\text { Radio- } \\
\text { thérapeute }\end{array}$ & $1 \mathrm{~h} 10 \mathrm{~min}$ & $1 \mathrm{~h}$ & $50 \mathrm{~min}$ & $40 \mathrm{~min}$ & $30 \mathrm{~min}$ & $40 \mathrm{~min}$ \\
\hline & Urologue & $30 \mathrm{~min}$ & $15 \mathrm{~min}$ & $15 \mathrm{~min}$ & $15 \mathrm{~min}$ & $15 \mathrm{~min}$ & $20 \mathrm{~min}$ \\
\hline
\end{tabular}




\subsubsection{Dose équivalente aux extrémités}

Les doses équivalentes aux extrémités enregistrées au cours des six curiethérapies, par les dosimètres nanoDot ${ }^{\circledR}$ placés au niveau des extrémités des opérateurs, sont également restées inférieures au seuil de détection de $100 \mu \mathrm{Sv}$.

\subsubsection{Dose équivalente au cristallin}

De même, les doses équivalentes à la peau mesurées au niveau du front des opérateurs par les dosimètres nanoDot ${ }^{\circledR}$ n'ayant pas dépassé le seuil de détection, nous estimons la dose équivalente au cristallin comme inférieure à $100 \mu \mathrm{Sv}$.

\subsection{Débits d'équivalent de dose mesurés}

La moyenne des débits mesurés au contact du porte source de $59 \mathrm{nSv} / \mathrm{h}$ reste dans le domaine du bruit de fond évalué à $62 \mathrm{nSv} / \mathrm{h}$.

Ceux mesurés au contact de l'extrémité de la cartouche pré-chargée (avec protection) sont de $2,88 \mu \mathrm{Sv} / \mathrm{h}$ en moyenne et s'élèvent à $4,67 \mathrm{mSv} / \mathrm{h}$ lorsque le grain d'iode est à nu (cartouche prête à l'insertion dans le « Mick applicator $\left.{ }^{\circledR} »\right)$.

Les débits d'équivalent de dose mesurés à partir du patient en différents points et temps de l'intervention sont donnés dans le tableau II.

\section{TABLEAU II}

Moyennes des mesures des débits d'équivalent de dose ambiant (min - max). Ambient dose equivalent rate measurements (min - max).

\begin{tabular}{|c|c|c|c|}
\hline & & $\begin{array}{c}\text { Fin d'implantation } \\
\text { des grains périphériques }\end{array}$ & $\begin{array}{l}\text { Fin d'intervention } \\
\text { (grains périphériques } \\
\text { et centraux en place) }\end{array}$ \\
\hline Pseudo contact du périnée & & $80 \mu \mathrm{Sv} / \mathrm{h}(33-145)$ & $170 \mu \mathrm{Sv} / \mathrm{h}(100-230)$ \\
\hline \multirow{2}{*}{ En direction des pieds } & à 20 cm* & $21 \mu \mathrm{Sv} / \mathrm{h} \quad(9,5-50)$ & $31 \mu \mathrm{Sv} / \mathrm{h}(15-53)$ \\
\hline & à 1 m* & $1,75 \mu \mathrm{Sv} / \mathrm{h} \quad(0,65-3,5)$ & $2,7 \mu \mathrm{Sv} / \mathrm{h} \quad(1,4-6)$ \\
\hline $\begin{array}{l}\text { En regard de l'abdomen } \\
\text { à } 20 \mathrm{~cm}^{*}\end{array}$ & & $9,4 \mu \mathrm{Sv} / \mathrm{h} \quad(3-17,5)$ & $23,7 \mu \mathrm{Sv} / \mathrm{h}(13,5-52)$ \\
\hline \multirow{2}{*}{ En direction de la tête } & à $20 \mathrm{~cm} *$ & $1,8 \mu \mathrm{Sv} / \mathrm{h}(0,8-3,5)$ & $8,6 \mu \mathrm{Sv} / \mathrm{h}(2,6-16)$ \\
\hline & à 1 m* & $0,14 \mu \mathrm{Sv} / \mathrm{h} \quad(0,06-0,34)$ & $0,2 \mu \mathrm{Sv} / \mathrm{h} \quad(0,07-0,45)$ \\
\hline \multirow{2}{*}{ Latéral } & à $20 \mathrm{~cm} *$ & mesure non réalisée & $6,6 \mu \mathrm{Sv} / \mathrm{h} \quad(0,5-30)$ \\
\hline & à 1 m* & $0,15 \mu \mathrm{Sv} / \mathrm{h} \quad(0,09-0,25)$ & $0,26 \mu \mathrm{Sv} / \mathrm{h} \quad(0,16-0,5)$ \\
\hline
\end{tabular}

* Mesures réalisées à la distance $\mathrm{X}(\mathrm{en} \mathrm{m}$ ou $\mathrm{cm}$ ) du site d'implantation (prostate) dans la direction indiquée. 


\subsection{Zonage radiologique}

Les doses efficaces susceptibles d'être reçues sur l'heure la plus pénalisante au niveau des différents postes de travail sont présentées dans le tableau III. Ces valeurs sont cohérentes avec les résultats de la dosimétrie individuelle.

Au regard des limites réglementaires, le poste de travail « opérateur » peut donc être classé en zone surveillée et les autres postes en zone non réglementée.

À partir de la dose la plus péjorative de $3,2 \mu \mathrm{Sv}$ mesurée à $30 \mathrm{~cm}$ du périnée au niveau du poste de travail de l'opérateur, la limite de la zone contrôlée est estimée par calcul à environ $20 \mathrm{~cm}$ du périnée du patient (puisque la limite entre ZS et ZC correspond à 7,5 $\mu \mathrm{Sv}$ en 1 heure).

En pratique, seules les extrémités (mains) du radiothérapeute et de l'urologue pénètrent en zone contrôlée.

\section{DISCUSSION}

\subsection{Analyse des résultats}

\subsubsection{Données générales}

Les résultats exprimés dans le tableau I démontrent clairement l'existence d'une courbe d'apprentissage sur six curiethérapies. Les temps d'intervention ont été réduits de moitié. Cette réduction significative se traduit également au niveau du temps d'exposition des opérateurs aux rayonnements ionisants. Le radiothérapeute, personnel le plus exposé, réduit en moyenne son temps d'exposition de 10 minutes par curiethérapie, passant ainsi d'une durée de 70 à 30 minutes d'exposition. L'urologue divise lui plus rapidement son temps d'exposition par deux, en abaissant son temps d'exposition de 30 à 15 minutes. Ces données sont en accord avec celles retrouvées dans la littérature (Lee et al., 2000). L'habileté et la rapidité des opérateurs augmentent avec le nombre de cas, particulièrement du fait de la familiarisation des gestes avec la technique d'implantation et l'utilisation du «Mick applicator ${ }^{\circledR} »$. Cette courbe d'apprentissage est un allié précieux en radioprotection.

\subsubsection{Dosimétrie des personnels}

Les doses cumulées lors des six curiethérapies étant restées en dessous du seuil de détection des dosimètres de $100 \mu \mathrm{Sv}$, les doses maximales susceptibles d'être 
reçues sont inférieures à $17 \mu \mathrm{Sv} /$ acte en $\mathrm{Hp}(10)$ et $\mathrm{Hp}(0,07)$. Ces résultats sont difficilement comparables puisque nous n'avons pas trouvé dans la littérature d'autres études dosimétriques portant sur une technique similaire à celle employée par notre équipe (technique en un seul temps utilisant un projecteur de source). Les seules données rapportées (données non publiées) sont celles de l'Institut Curie de Paris pour lesquelles les doses équivalentes enregistrées par une bague TLD portée à chaque main et lue après six curiethérapies sur un mois, restent également inférieures au seuil de détection de $100 \mu \mathrm{Sv}$. Ces résultats semblent donc confirmer nos mesures.

En revanche, les publications relatives à l'exposition radiologique de l'équipe opératoire au cours de curiethérapies de prostate faisant appel à une technique différente sont plus nombreuses. Ces publications concernent une méthode dite de «preplanning » utilisant la fluoroscopie. Pour cette technique, après une première phase précise de planification, les aiguilles sont implantées dans une seconde phase en adhérant strictement à ce qui a été planifié. Le respect de la position exacte des aiguilles nécessite souvent l'emploi de la fluoroscopie (van Haaren et al., 2010). Classiquement, cette technique repose sur l'implantation manuelle d'aiguilles pré-chargées avec des grains liés dans un matériel de suture type « rapid-strands ${ }^{\circledR} »$.

Sur 24 curiethérapies, Schiefer et al. (2009) retrouvent avec des dosimètres TLD une dose équivalente de $1,31 \mathrm{mSv}$ au niveau des doigts et $0,61 \mathrm{mSv}$ au niveau des mains chez des radiothérapeutes expérimentés (contre 2,07 $\mathrm{mSv}$ et $1,05 \mathrm{mSv}$ pour des débutants), autorisant la réalisation de moins de 400 actes par an sans dépasser la limite réglementaire de $500 \mathrm{mSv}$. Anglesio et al. (2005) enregistrent eux sur 60 curiethérapies, avec un dosimètre corps entier et une bague TLD, une dose efficace inférieure à $30 \mu \mathrm{Sv}$ et une dose équivalente de $420 \mu \mathrm{Sv}$ par acte. Pour Kaulich et al. (2002) la dose équivalente aux extrémités peut atteindre $1 \mathrm{mSv}$.

Schwartz et al. (2003) ont montré que le temps de fluoroscopie était bien le principal facteur d'exposition. L'utilisation de la fluoroscopie contribue ainsi à délivrer à l'organisme entier une dose efficace estimée à 7,6 $\mu \mathrm{Sv} / \mathrm{min}$ et une dose équivalente aux extrémités de $50 \mu \mathrm{Sv} / \mathrm{min}$.

Nous rappelons que dans notre étude, l'hypothèse enveloppe retenue aux vues des résultats, évalue les doses maximales efficaces et équivalentes aux extrémités à $17 \mu$ Sv/acte. 


\subsubsection{Débits d'équivalent de dose}

L'originalité de notre étude réside dans la mesure des débits directement au niveau du périnée du patient, en per et post-opératoire immédiat, rarement évalués dans d'autres études. Les valeurs retrouvées sont relativement importantes puisque de l'ordre de $170 \mu \mathrm{Sv} / \mathrm{h}$ au contact, $31 \mu \mathrm{Sv} / \mathrm{h}$ à $20 \mathrm{~cm}$ et $2,7 \mu \mathrm{Sv} / \mathrm{h}$ à $1 \mathrm{~m}$ (Tab. II). Ces débits instantanés, mesurés en fin d'intervention, sont à différencier des valeurs retrouvées après intégration sur l'heure la plus pénalisante au niveau des différents postes de travail (Tab. III).

En revanche, il est possible de comparer nos valeurs à celles de la littérature pour les débits de dose mesurés à $20 \mathrm{~cm}$ au niveau de la surface antérieure de l'abdomen et à $1 \mathrm{~m}$ sur le côté du patient. Ainsi, le débit d'équivalent de dose moyen de $23,7 \mu \mathrm{Sv} / \mathrm{h}$ mesuré à $20 \mathrm{~cm}$ en avant de l'abdomen est concordant avec les moyennes retrouvées dans d'autres études : $15,9 \mu \mathrm{Sv} / \mathrm{h}$ pour Cattani et al. (2006) et $22 \mu \mathrm{Sv} / \mathrm{h}$ pour l'Institut Curie (IRSN, 2003). Le débit de dose latéral à $1 \mathrm{~m}$ estimé dans notre étude à $0,25 \mu \mathrm{Sv} / \mathrm{h}$ est du même ordre de grandeur que les $0,3 \mu \mathrm{Sv} / \mathrm{h}$ retrouvés par Smathers et al. (1999) et les 0,9 $\mu \mathrm{Sv} / \mathrm{h}$ pour Dauer et al. (2004).

Les débits de dose mesurés à distance du patient dépendent du nombre de grains d'iode et de l'activité totale implantés, de la distribution géographique des grains, de la position de la prostate et de l'épaisseur de tissus traversée.

\section{TABLEAU III}

Doses efficaces intégrées en 1 heure aux différents postes de travail. Effective doses integrated in 1 hour with different workstations.

\begin{tabular}{|c|c|c|}
\hline $\begin{array}{l}\text { Poste de travail } \\
\text { (distance / périnée) }\end{array}$ & Résultats & Classement de la zone \\
\hline $\begin{array}{c}\mathrm{N}^{\circ} 1: \text { mesure à } 30 \mathrm{~cm} \\
\text { "Opérateur " }\end{array}$ & $\begin{array}{c}3,20 \mu \mathrm{Sv} \\
\text { (soit } 128 \mu \mathrm{Sv} \text { sur } 1 \text { mois)* }\end{array}$ & Zone surveillée \\
\hline $\begin{array}{l}\mathrm{N}^{\circ} 2: \text { mesure à } 80 \mathrm{~cm} \\
\text { "Écran de l'échographe" }\end{array}$ & $\begin{array}{c}0,60 \mu \mathrm{Sv} \\
\text { (soit } 24 \mu \mathrm{Sv} \text { sur } 1 \text { mois)* }^{*}\end{array}$ & Zone non réglementée \\
\hline $\begin{array}{l}\mathrm{N}^{\circ} 3: \text { mesure à } 50 \mathrm{~cm} \\
\text { "Table d'instrumentation" }\end{array}$ & $\begin{array}{c}1,00 \mu \mathrm{Sv} \\
\text { (soit } 40 \mu \mathrm{Sv} \text { sur } 1 \text { mois)* }\end{array}$ & Zone non réglementée \\
\hline $\begin{array}{l}\mathrm{N}^{\circ} 4: \text { mesure à } 150 \mathrm{~cm} \\
\text { "Pupitre radiophysicien" }\end{array}$ & $\begin{array}{c}0,19 \mu \mathrm{Sv} \\
\text { (soit 7,6 } \mu \mathrm{Sv} \text { sur } 1 \mathrm{mois})^{*}\end{array}$ & Zone non réglementée \\
\hline $\begin{array}{l}\mathrm{N}^{\circ} 5: \text { mesure à } 160 \mathrm{~cm} \\
\text { "Écrans monitorage IADE" }\end{array}$ & $\begin{array}{c}0,10 \mu \mathrm{Sv} \\
\text { (soit } 4 \mu \mathrm{Sv} \text { sur } 1 \text { mois)* }\end{array}$ & Zone non réglementée \\
\hline
\end{tabular}

* Valeurs calculées sur la base de 40 heures de travail mensuel. 


\subsubsection{Limites de l'étude}

Comme le souligne la CIPR dans sa publication 98 (ICRP, 2005), la mesure de doses aussi basses, délivrées à bas débit par des photons de basses énergies se révèle difficile. Ainsi, malgré la sélection d'appareils adaptés en énergie et en sensibilité et la réalisation des mesures par des équipes expérimentées, nous pensons que les résultats présentés ne sont qu'une estimation de la réalité de l'exposition. Nous savons, d'après cette même publication, que généralement certains débits mesurés par le radiamètre peuvent être surestimés et qu'à l'inverse, les doses enregistrées par les dosimètres peuvent être sous-évaluées (dosimétrie opérationnelle notamment). Néanmoins en pratique, ces incertitudes n'ont aucune conséquence pour la radioprotection des personnels. Dans un but scientifique, il conviendrait d'étudier avec plus de précision les courbes de réponse de ces détecteurs dans les basses énergies, en dose et en direction, afin d'appliquer le cas échéant les facteurs correctifs nécessaires.

\subsection{Recommandations}

Les résultats de cette étude de poste nous permettent de proposer des recommandations pour le zonage radiologique, la surveillance dosimétrique et la catégorisation des personnels.

\subsubsection{Zonage radiologique et surveillance dosimétrique}

Pour l'acte de curiethérapie, la zone contrôlée s'étend sur une sphère de $20 \mathrm{~cm}$ de rayon, centrée sur la prostate du patient. En pratique, la limite de cette zone réglementée n'est pas physiquement matérialisable.

Pour ce qui concerne la limite de la zone surveillée, nous estimons préférable de l'étendre aux parois de la salle opératoire compte tenu d'un risque toujours possible d'incident tel qu'un blocage dans le projecteur de source, voire la perte de grains. La signalisation par le trèfle de cette zone réglementée est ainsi facilitée à l'entrée de la salle et donc mieux identifiée par l'ensemble des personnels du bloc opératoire.

Compte tenu de ces éléments, nous recommandons le port d'un dosimètre passif pour les opérateurs (urologue et radiothérapeute). Concernant la surveillance dosimétrique des extrémités, il convient de poursuivre les mesures sur une période de 6 à 12 mois en activité «normale » de curiethérapie. La décision du maintien ou non de cette surveillance sera alors fonction des doses enregistrées sur deux à quatre périodes de trois mois. Si, comme dans notre étude, les doses restent inférieures au seuil de détection, il sera alors licite de ne pas poursuivre cette surveillance. 
La dosimétrie opérationnelle n'est pas nécessaire puisque seules les mains de ces opérateurs pénètrent en zone contrôlée.

Par ailleurs, la surveillance dosimétrique des IBODE ET IADE n'est pas obligatoire.

\subsubsection{Catégorisation des personnels}

Les doses maximales « corps entier » en Hp (10) susceptibles d'être reçues par les opérateurs (urologue et radiothérapeute) étant inférieures à $100 \mu \mathrm{Sv}$ pour 6 actes, la limite annuelle de dose efficace pour le public fixée à $1 \mathrm{mSv}$ en dose efficace pourrait donc être atteinte après 60 curiethérapies par an, ce qui est envisageable.

Au vue de la définition du travailleur exposé ${ }^{6}$ (circulaire DGT/ASN n ${ }^{\circ} 04 \mathrm{du}$ 21 avril 2010), nous recommandons de classer les opérateurs (urologue et radiothérapeute) comme personnels exposés de catégorie B. Ces personnels doivent donc bénéficier d'une surveillance médicale renforcée associée à une surveillance dosimétrique adaptée.

L'IBODE et l'IADE, personnels non rattachés de manière habituelle à cette activité de curiethérapie (personnels tournants), peuvent être considérés comme des travailleurs non classés accédant occasionnellement à une zone réglementée. En effet, comme défini dans cette même circulaire, «un travailleur dont l'intervention ne modifie pas notablement les conditions d'exposition peut accéder de manière occasionnelle à une zone réglementée sans être classé ni faire l'objet d'un suivi dosimétrique de référence si l'employeur :

- a évalué préalablement les doses susceptibles d'être reçues»: ce qui est l'objet de notre étude ;

- "s'est assuré que leur cumul avec d'autres doses éventuellement préalablement reçues demeure inférieur à $1 \mathrm{mSv}$ sur les douze derniers mois glissants » : ce qui sera vérifié par la personne compétente en radioprotection et le médecin du travail ;

- «a mesuré les doses effectivement reçues lorsque ce travailleur intervient en zone contrôlée au moyen notamment d'une dosimétrie opérationnelle » : nous avons vu précédemment que cela n'est pas nécessaire.

En revanche, le radiophysicien est déjà catégorisé $B$ et son activité en curiethérapie ne justifie pas un classement supérieur.

\footnotetext{
6 Est considéré comme travailleur exposé tout travailleur susceptible de dépasser, dans le cadre de son activité professionnelle, l'une des valeurs limites de dose fixées pour le public, quelles que soient les conditions de réalisation de l'opération ; habituelles ou bien liées à un incident.
} 
Toutefois ces recommandations ne sont données qu'à titre indicatif puisque la définition de la catégorisation et du zonage est de la responsabilité de l'employeur.

\subsubsection{Réflexions concernant le port d'équipements de protection individuelle (EPI)}

Compte tenu des résultats, le port d'EPI (tabliers plombés, gants de protection radiologique, cache-thyroïde) est inutile au cours de ce type d'intervention. En revanche, lors de la réalisation d'un cliché radiologique de contrôle, le port du tablier plombé pour les personnels qui doivent être présents dans la pièce est obligatoire.

\subsubsection{Radioprotection vis-à-vis du patient}

Concernant l'environnement autour du patient, les débits de dose mesurés en fin d'intervention sont de $170 \mu \mathrm{Sv} / \mathrm{h}$ au pseudo-contact du périnée et de $23,7 \mu \mathrm{Sv} / \mathrm{h}$ à $20 \mathrm{~cm}$ en regard de l'abdomen. À ces distances, il faudrait être présent pendant 6 et 40 heures pour dépasser la limite annuelle publique de $1 \mathrm{mSv}$. Ce scénario n'est en pratique pas envisageable. La présence du patient ne pose donc pas de problème particulier d'exposition externe vis-à-vis des autres patients et personnels en salle de réveil, comme en unité d'hospitalisation.

\subsection{Une technique peu irradiante mais très encadrée}

La curiethérapie de prostate par implants permanents de grains d'iode libres implantés par un applicateur, réalisée en un temps par voie transpérinéale échoguidée, est une technique peu irradiante pour les personnels.

L'exposition radiologique de l'équipe opératoire est faible du fait de plusieurs facteurs :

- la source radioactive d'iode-125, émettrice de photons de basses énergies (30 keV en moyenne) ;

- l'atténuation par les tissus péri-prostatiques puisque la couche de demiatténuation est estimée à environ $3 \mathrm{~cm}$;

- l'atténuation du faisceau résiduel par la grille de repérage en matière plastique dense ;

- la technique elle-même : radioprotection des opérateurs optimisée par la cartouche en acier dans laquelle sont stockés les grains d'iode qui transitent entre un support plombé et le projecteur de source ;

- pas d'utilisation de scopie. 
Néanmoins, la mise en œuvre de cette technique implique d'importantes contraintes en termes d'information du patient et d'organisation, prises en compte à l'HIA du Val-de-Grâce dans une note d'information destinée aux patients et par le biais de procédures standardisées :

- Le patient doit être porteur d'une carte personnelle indiquant la date de la curiethérapie et le nom de l'hôpital où elle a été réalisée. Ces informations peuvent s'avérer utile en cas de chirurgie ouverte abdomino-pelvienne ou lors $\mathrm{du}$ franchissement de détecteurs particulièrement sensibles dans certains aéroports. Il doit être averti de l'impossibilité de recourir à l'incinération postmortem dans un délai de 3 ans après l'implantation (risque de contamination des personnels à l'iode par les fumées) et éviter, dans un souci de respect du principe ALARA, de prendre sur les genoux des nourrissons et des enfants de moins de 13 ans et se tenir à distance des femmes enceintes au cours des deux mois suivant l'intervention.

- Les mesures organisationnelles sont centrées sur le risque de perte de grains afin d'éviter une éventuelle contamination. Elles comprennent :

- une gestion sans faille des sources radioactives avant (traçabilité) et pendant (comptage des grains) l'implantation ;

- un contrôle radiologique de la table d'instrumentation en cours d'intervention, des déchets opératoires en fin d'implantation et lors de la réfection du pansement périnéal en unité d'hospitalisation ;

- une filtration des urines pendant 15 jours, deux semaines d'abstinence suivies de rapports protégés avec préservatifs pendant encore 15 jours, avec le cas échéant, récupération du grain perdu à l'aide d'une cuillère ou d'une pince à épiler dans le container plombé remis lors de la sortie d'hospitalisation (repris par le service d'oncologie-radiothérapie).

\section{CONCLUSION}

L'étude de poste est imposée par la réglementation (code du travail) et en est l'un des éléments principaux ${ }^{7}$ (IRSN, 2010). Elle intervient pour la délimitation des zones réglementées (article R. 4451-18) et la catégorisation des travailleurs (articles R. 4451-44 à R. 4451-46). Les choix faits permettent de statuer sur le suivi dosimétrique individuel des personnels exposés. C'est un élément essentiel pour s'assurer du respect des limites réglementaires et du principe d'optimisation de la radioprotection (article R. 4451-10). L'étude de poste relève de la responsabilité de l'employeur, qui en confie la réalisation à la personne compétente en

\footnotetext{
7 Article R. 4451-11 du code du travail : «Dans le cadre de l'évaluation des risques, l'employeur [...] procède à une analyse des postes de travail qui est renouvelée périodiquement et à l'occasion de toute modification des conditions pouvant affecter la santé et la sécurité des travailleurs. [...]».
} 
radioprotection. Enfin, les résultats de l'étude de poste interviennent pour renseigner la fiche d'exposition établie par l'employeur (article R. 4451-57) et utilisée par le médecin du travail (article R. 4451-82) qui statue sur l'aptitude médicale et les modalités de surveillance médicale de chaque travailleur.

Cette étude dosimétrique apporte des résultats fiables et propose des recommandations aux différents acteurs de la radioprotection dans le cadre particulier de l'activité de curiethérapie de prostate. Elle montre que la curiethérapie de prostate par implants permanents d'iode-125, réalisée en temps réel par voie transpérinéale au moyen d'un applicateur, est une technique peu irradiante pour le personnel.

Si le risque d'exposition externe peut apparaître comme faible, la mise en œuvre de cette technique implique néanmoins d'importantes mesures organisationnelles afin de respecter les grands principes de radioprotection: justification, optimisation et limitation.

Cette étude constitue assurément un outil de communication et d'information pour les personnels travaillant au bloc opératoire, plus habitués à la présence de générateurs de rayons $\mathrm{X}$ qu'à celle de sources radioactives scellées.

Remerciements. Les auteurs remercient chaleureusement pour leur participation active à cette étude les équipes de radiothérapie, d'urologie et du bloc opératoire de l'Hôpital d'instruction des armées du Val-de-Grâce, dirigées par le médecin général inspecteur Debonne, ainsi que mademoiselle Noëlle Pierrat, physicienne médicale à l'Institut Curie de Paris pour son concours.

\section{RÉFÉRENCES}

Anglesio S., Calamia E., Fiandra C., Giglioli FR., Ragona R., Ricardi U., Ropolo R. (2005) Prostate brachytherapy with iodine-125 seeds: radiation protection issues, Tumori 91, 335-338.

Arrêté du 15 mai 2006 relatif aux conditions de délimitation et de signalisation des zones surveillées et contrôlées et des zones spécialement réglementées ou interdites compte tenu de l'exposition aux rayonnements ionisants, ainsi qu'aux règles d'hygiène, de sécurité et d'entretien qui y sont imposées, Journal officiel de la République Française du 15 juin 2006.

Cattani F., Vavassori A., Polo A., Rondi E., Cambria R., Orecchia R., Tosi G. (2006) Radiation exposure after permanent prostate brachytherapy, Radiotherapy and Oncology 79, 65-69.

Circulaire DGT/ASN n 04 du 21 avril 2010 relative aux mesures de prévention des risques d'exposition aux rayonnements ionisants.

Code du travail. Partie réglementaire nouvelle - Quatrième partie - LIVRE IV - Titre V - Chapitre $1^{\mathrm{er}}$ : prévention des risques d'exposition aux rayonnements ionisants.

Cosset J.M., Haie-Meder C. (2005) Curiethérapie du cancer prostatique : haut débit ou bas débit de dose ? Cancer/Radiothérapie 9, 610-619.

Cosset J.M., Pinillos-Ashton L., Mettler F. (2004) Recommandations de la Commission international de protection radiologique (CIPR) pour la curiethérapie de haut débit de dose et la curiethérapie de prostate, Cancer/Radiothérapie 8, S50-S55. 


\section{G. GAGNA et al.}

Dauer L.T., Zelefsky M., Horan C. (2004) Assessment of radiation safety instructions to patients based on measured dose rates following prostate brachytherapy, Brachytherapy 3, 1-6.

ICRP Publication 98 (2005) Radiation safety aspects of brachytherapy for prostate cancer using permanently implanted sources, Ann. ICRP 35(3).

IRSN (2003) Report SDOS 2003-2009, Institut de radioprotection et de sûreté nucléaire.

IRSN (2010) Guide pratique. Réalisation des études dosimétriques de poste de travail présentant un risque d'exposition aux rayonnements ionisants (version 2), DPRH/DIR n ${ }^{\circ} 2010-1$.

Kaulich T.W., Lamprecht U., Paulsen F., Lorenz J., Maurer U., Loeser W., Bichler KH., Nüsslin F., Bamberg M. (2002) Physical and technical quality assurance and radiation protection in transperineal interstitial permanent prostate brachytherapy with 125-iodine seeds, Strahlenther Onkol. 178, 667-675.

Lee WR., deGuzman AF., Bare RL., Marshall MG., McCullough DL. (2000) Postimplant analysis of transperineal interstitial permanent prostate brachytherapy: evidence for a learning curve in the first year at a single institution, Int. J. Radiat. Oncol. Biol. Phys. 46, 85-88.

Peiffert D. (2005) Curiethérapie interstitielle des cancers localisés de prostate par implants permanents d'iode 125, Cancer/Radiothérapie 9, 388-393.

Schiefer H., von Toggenburg F., Seelentag W., Plasswilm L., Ries G., Lenggenhager C., Schmid H.P., Leippold T., Prikler L., Krusche B., Roth J., Engeler D. (2009) Exposure of treating physician to radiation during prostate brachytherapy using iodine-125 seeds: dose measurements on both hands with thermoluminescence dosimeters, Strahlenther Onkol. 185, 689-695.

Schwartz D.J., Davis B.J., Vetter R.J., Pisansky T.M., Herman M.G., Wilson T.M., LaJoie W.N., Oberg A.L. (2003) Radiation exposure to operating room personnel during transperineal interstitial permanent prostate brachytherapy, Brachytherapy 2, 98-102.

Smathers S., Wallner K., Korssjoen T., Bergsagel C., Hudson R.H., Sutlief S., Blasko J. (1999) Radiation safety parameters following prostate brachytherapy, Int. J. Radiat. Oncol. Biol. Phys. 45, 397-399.

van Haaren PM., van't Riet A., Moerland MA., Koedooder C., Westendorp H. (2010) Dose to fingertips of staff preparing stranded iodine-125 seeds for permanent prostate implants, Radiat. Prot. Dosim. (article accepté le 20 octobre 2010, en attente de publication).

Wassilieff S. (2009) Cataracte et rayonnements ionisants, Radioprotection 44, 505-517. 